\title{
ESTADOS HIPERANDROGÉNICOS: REVISIÓN DE LA LITERATURA
}

\section{Hyperandrogenic states: a literature review}

\author{
Gustavo Alfonso Villarreal-Tordecilla, M.D.*
}

Recibido: junio 12/09 - octubre 26/09

\section{RESUMEN}

Objetivos: realizar una revisión de la fisiopatología, la etiología, el cuadro clínico, el diagnóstico y el tratamiento de los estados hiperandrogénicos.

Metodología: se realizó una búsqueda electrónica en PubMed/Medline para recopilar información publicada tanto en inglés como en español desde el año 1990 a 2009. Después, se eligieron artículos de investigación en hiperandrogenismo que evaluaran la fisiopatología, la etiología, el cuadro clínico, el diagnóstico y el tratamiento de los estados hiperandrogénicos, usando finalmente resúmenes para llevar a cabo el análisis.

Resultados: el exceso de andrógenos se puede producir por una alteración en el transporte, en la respuesta final, por el bloqueo de su transformación en estrógenos y por la administración exógena. Asimismo, éstos tienen su origen principalmente en los ovarios y las glándulas suprarrenales, expresándose clínicamente en tres aspectos diferentes: unidad pilosebácea y desórdenes reproductivos y metabólicos. Estos estados repercuten negativamente a nivel estético, reproductivo, metabólico y cardiovascular, por lo cual es necesario realizar un perfil hormonal y estudios de imagen con el fin de obtener un diagnóstico. Además, en algunas entidades existe un tratamiento específico,

* Especialista en Medicina Interna, Universidad Nacional de La Plata (Argentina). Fellow $2^{\text {do }}$ año de Endocrinología y Metabolismo. Servicio de Ginecología Endocrinológica, Hospital Militar Central. Bogotá (Colombia).Correo electrónico: gusalvitor@hotmail.com mientras que en otras el manejo está orientado a la sintomatología predominante.

Conclusiones: la fisiopatología y la etiología del exceso de andrógenos son muy variadas. Debido al cuadro clínico y a las consecuencias que esta entidad genera, se requiere estudio y manejo multidisciplinario.

Palabras clave: hiperandrogenismo, resistencia a la insulina.

\section{SUMMARY}

Objectives: reviewing the pathophysiology, aetiology, clinical features, diagnosis and treatment of hyperandrogenic states.

Methodology: an online search was carried out of literature in PubMed/Medline, published in English or Spanish from 1990 to 2009 regarding research articles, assessing the pathophysiology, aetiology, clinical features, diagnosis and treatment of hyperandrogenic states. Their summaries were then used for analysing them.

Results: androgen excess may result from changes in androgen transportation, blockage of androgen conversion into estrogens, altered final response and through exogenous administration. It mostly originates in the ovaries and adrenals and is clinically expressed at the pilosebaceous unit, reproductive and metabolic levels. These states have a negative aesthetic, reproductive, metabolic and/or cardiovascular impact. Proper diagnosis requires a hormone profile plus imaging studies. Some organisations provide specific treatment, while others rather focus on the predominant symptoms. 
Conclusions: excess androgen's pathophysiology and aetiology vary widely, and the clinical picture and consequences it produces require a multidisciplinary approach for both its study and management.

Key words: hyperandrogenism, insulin resistance.

\section{INTRODUCCIÓN}

Los estados hiperandrogénicos (EHA) son entidades clínicas que cursan con un exceso de andrógenos (ADG). Estos estados son un motivo de consulta muy frecuente en las clínicas ginecológicas y dermatológicas. Se producen por múltiples patologías, principalmente a nivel de los ovarios y las glándulas suprarrenales; no obstante, algunas endocrinopatías, como el hipotiroidismo y la hiperprolactinemia (HPRL) también pueden cursar con hiperandrogenismo.

Entre los variados mecanismos fisiopatológicos se incluyen: alteración en el transporte, bloqueo de su transformación en estrógenos, alteración en la respuesta final y administración de ADG exógenos. ${ }^{1}$ El EHA más recurrente es el Síndrome de Ovario Poliquístico (SOP), por lo tanto, son los ginecólogos quienes diagnostican esta alteración con mayor frecuencia; pero más allá de la oligo-anovulación e infertilidad, el aumento de abortos espontáneos y el mayor riesgo de cáncer de endometrio, también se encontraron otras manifestaciones clínicas en la unidad pilosebácea así como alteraciones endocrino-metabólicas. ${ }^{2,3}$

Del mismo modo, la frecuencia de los EHA es muy variable. Ésta es baja cuando es evaluada aisladamente, donde puede incluso no advertirse ningún signo, pero cuando se unifican todas las consecuencias generales se observa claramente que es más frecuente de lo que se sabe. Por esta razón, el abordaje general es muy importante debido a las complicaciones que se presentan. Una de las complicaciones más importantes es la relación existente entre la resistencia a la insulina (RI), el desarrollo de diabetes mellitus tipo 2 y el síndrome metabólico. ${ }^{2,4,5}$ Ahora bien, la coexistencia de obesidad tiene una responsabilidad fisiopatológica adicional, no sólo en el desarrollo de la RI y sus anomalías metabólicas asociadas, sino también en el hiperandrogenismo y sus consecuencias clínicas acompañantes, como los trastornos menstruales y la infertilidad. ${ }^{6}$

Asimismo, hay que enfatizar en el estudio y el manejo multidisciplinario debido a las diversas manifestaciones clínicas y complicaciones generadas. Entre éstas, llama mucho la atención las alteraciones endocrino-metabólicas y; por consiguiente, el riesgo cardiovascular, de allí que en su manejo se involucre a profesionales que van desde enfermeras hasta personal de las diversas especialidades médicas de acuerdo con las manifestaciones clínicas presentes. Por lo tanto, y con el objetivo de revisar aspectos relacionados con la fisiopatología, la etiología, las implicaciones clínicas, el diagnóstico y el tratamiento de los EHA, se hizo una revisión de la literatura pertinente.

\section{METODOLOGÍA}

Estrategia de búsqueda: utilizando las palabras claves, "hiperandrogenismo" y "resistencia a la insulina", se realizó una búsqueda electrónica en PubMed/ Medline publicada en inglés o español desde el año 1990 a 2009.

Criterios de selección: se eligieron artículos de investigación en hiperandrogenismo que evaluaran la fisiopatología, la etiología, el cuadro clínico, el diagnóstico y el tratamiento de la entidad. De esta manera, la información fue revisada y finalmente resumida.

\section{RESULTADOS}

Entre 150 artículos obtenidos en PubMed/ Medline, se evaluaron finalmente 61 artículos que respondieron con los objetivos planteados.

\section{Fisiopatología}

El exceso de ADG se puede presentar en cinco circunstancias distintas. ${ }^{1}$

1. Aumento en la producción endógena: secreción directa por gónadas y/o suprarrenales o conversión periférica. 
2. Alteración en el transporte: la disminución de la globulina transportadora de hormonas sexuales (HSBG) incremeta los niveles de ADG en forma libre.

3. Bloqueo de su transformación en estrógenos: carencia de la enzima p-450 aromatasa.

4. Alteración en la respuesta final: aumento de la actividad enzimática de 5 alfa reductasa y/o aumento en la sensibilidad del receptor de andrógenos (RA).

5. Administración de ADG exógenos.

El exceso de ADG se expresa clínicamente en tres niveles: unidad pilosebácea, sistema reproductivo y en el metabolismo de lípidos-carbohidratos; por ende, se presenta un mayor riesgo de enfermedad cardiovascular. A continuación, presentamos un comentario sobre este último punto. ${ }^{7-9}$

\section{Trastornos metabólicos e hiperandrogenismo}

La RI no es un hallazgo universal. Por ejemplo, De Ugarte y demás colegas la encontraron en 60\% de las pacientes con SOP por medio del Homeostasis Model Assesment (HOMA, por sus siglas en inglés). Incluso algunas de ellas no desarrollan SOP pese a presentar una RI marcada. Del mismo modo, la evidencia sugiere que el hiperandrogenismo en etapas tempranas de la vida, e incluso antes del nacimiento, determina la distribución de la grasa con patrón androide en algunas mujeres, lo que predispone a la RI y a sus complicaciones metabólicas.

En este sentido, en los EHA existe una disminución en las concentraciones de adiponectina (la adipocina favorece la sensibilidad insulínica) con una distribución androide de la grasa abdominal, independiente de la obesidad. ${ }^{10,11}$

Por lo tanto, podría existir un círculo vicioso, según el cual el hiperandrogenismo podría facilitar la disposición anormal de grasa, en parte mediada por la disminución de la adiponectina y en otra parte por la secreción del tejido adiposo de otras moléculas como citocinas inflamatorias, e inducir la RI, el hiperinsulinismo endógeno y el agravamiento del hiperandrogenismo. ${ }^{12,13}$ El resultado final de la hiperinsulinemia es el favorecimiento hacia las altas concentraciones de ADG por múltiples mecanismos: estimulación de la secreción por el ovario, aumento en la expresión de receptores del factor de crecimiento similar a la insulina (IGF) en el ovario, aumento en la síntesis de ADG suprarrenales en respuesta a la corticotropina $(\mathrm{ACTH})$, aumento en los receptores de la hormona luteinizante (LH) en los ovarios, aumento en la respuesta de la $\mathrm{LH}$ a la gonadoliberina $(\mathrm{GnRH})$ en la hipófisis ${ }^{14-16}$ y disminución de la síntesis hepática de SHBG y de la proteína ligadora del factor de crecimiento afín a la insulina-1(IGFBP-1) en el hígado. ${ }^{17-19}$ Ahora bien, como resultado final de la disminución del IGFBP-1 se produce un aumento en las concentraciones del de IGF-1 y una mayor actividad local del IGF-1 e IGF-2 en el ovario. A su vez, la mayor actividad del IGF-1 en el endometrio y la activación directa por la insulina de los receptores del IGF o de su propio receptor son mecanismos posibles para el crecimiento endometrial (y el mayor riesgo de cáncer endometrial en estos estados). ${ }^{20,21}$

\section{Etiología}

Son muchas las entidades que cursan con un exceso de ADG. Para facilitar reconocerlas, se recomienda clasificarlas de acuerdo al origen. Tabla 1. Entre éstas caben destacar el SOP, sobre el cual se dice que con el tiempo podría considerarse un problema de salud pública para el mundo contemporáneo. ${ }^{22,23}$

Igualmente, la disposición de la grasa con patrón androide lleva a un notable aumento de enfermedades cardiovasculares y diabetes mellitus tipo 2. ${ }^{24-27}$

En el caso del cociente triglicéridos/colesterol HDL, éste se podría considerar un método útil y práctico para identificar el riesgo cardiovascular aumentado en pacientes con SOP, el cual está estrechamente relacionado con el perímetro abdominal y los índices de resistencia y sensibilidad a la insulina. ${ }^{28-30}$ Asimismo, se ha demostrado que la grasa visceral está asociada con enfermedad cardiovascular subclínica en pacientes con SOP. ${ }^{31-33}$ 


\section{Tabla 1. Principales estados hiperandrogénicos.}

\section{OVÁRICAS}

SOP

Hipertecosis ovárica

Tumores secretores de andrógenos

\section{SUPRARRENALES}

Hiperplasia suprarrenal congénita de comienzo tardío

Adenomas - carcinomas

\section{Manifestaciones clínicas}

Los EHA se manifiestan por medio de hirsutismo, acné, seborrea y/o alopecia de patrón masculino, además de oligo-anovulación, infertilidad, y manifestaciones clásicas de insulino-resistencia. ${ }^{10,24,28,34}$

\section{Diagnóstico}

El registro de una buena historia clínica equivale a realizar exitosamente un correcto diagnóstico.

- Anamnesis: este examen clínico es de gran importancia por los antecedentes personales, la evolución del proceso y los tratamientos realizados anteriormente. ${ }^{35,36}$ Existen tres situaciones que obligan a los médicos a hacer el diagnóstico lo más rápido posible: el síndrome de Cushing, por la elevada morbilidad y posibilidad de un carcinoma suprarrenal; los niveles de testosterona extremadamente elevados y la rápida evolución de los síntomas, ya que orientan hacia la presencia de un tumor. ${ }^{37,38}$

- Examen físico: se debe cuantificar el hirsutismo mediante la escala de Ferriman-Galwey, ${ }^{39,40}$ el grado de obesidad, los signos de virilización, la palpación de masas abdominales, la galactorrea, la fascie cushinoide, los rasgos acromegálicos e hipotiroideos, etc. ${ }^{35,36,38}$

- Bioquímica: debe incluir LH, FSH, prolactina, 17-hidroxi-progesterona, androstenediona, testosterona y sulfato de dehidroepiandrosterona. Cuando las pruebas basales no son conclusivas, se debe recurrir a los test dinámicos como el test de supresión con dexametasona, en busca del síndrome de Cushing; el test de estimulación con ACTH para hiperplasia suprarrenal congénita; y finalmente el test de frenación ovárica utilizando un estrógeno más progestágenos o análogos cuando las cifras de testosterona son demasiado altas. $^{36,38}$

Además, las imágenes ayudan a localizar el origen de la alteración. En tal caso, se pueden realizar ecografías, resonancia nuclear magnética, tomografía axial computada y gammagrafías. ${ }^{36}$

\section{Tratamiento}

El enfoque estará determinado por la clínica en torno a: exceso de ADG/oligo-anovulación, insulinoresistencia, hirsutismo, obesidad y riesgos conexos e infertilidad y manejo de alteraciones psico-afectivas. ${ }^{41}$ A lo anterior, existen entidades que tienen un tratamiento específico. Por ejemplo, cirugías y eventualmente radioterapia y/o quimioterapia para los tumores de ovario o suprarrenales, los protocolos de manejo en caso de hipotiroidismo, hiperprolactinemia, síndrome de Cushing, etc. ${ }^{42,43}$ Por ser el SOP el EHA más frecuente, a continuación se detalla el manejo de acuerdo con las anteriores pautas.

\section{Sintomatología dependiente del exceso de andrógenos/oligo- anovulación}

- Progestágenos en la segunda mitad del ciclo: tienen la capacidad de inhibir las gonatrofinas, y por tanto, se presenta una menor secreción de la LH. El resultado final es un menor estímulo de ésta sobre las células de teca y una menor síntesis de ADG. A nivel endometrial, frena el efecto mitógeno de los estrógenos. Se puede utilizar $10 \mathrm{mg}$ de medroxiprogesterona o 200 mg de progesterona micronizada. ${ }^{41,44}$

- Combinación de estrógenos y progestágenos: además de los efectos del progestágeno, el componente estrogénico es útil para incrementar la síntesis hepática de SHBG, con lo cual disminuyen las concentraciones de estrógenos y ADG libres. 
Se administran en forma de anticonceptivos orales. ${ }^{41,44,45}$

\section{Resistencia a la insulina, hirsutismo y riesgos conexos}

- Metformina: actúa incrementando la sensibilidad periférica a la insulina. ${ }^{46,47}$ La evidencia ha demostrado que el uso de metformina en forma aislada da lugar a la regularización de los ciclos. Igualmente, puede usarse en asocio con el clomifeno y con la FSH recombinante, mejorando las tasas de ovulación y concepción. ${ }^{48,49}$

Inicialmente, la metformina puede aumentar la hiperpulsatibilidad de la LH como mecanismo compensatorio regulador, y al mismo tiempo, reducir los niveles de insulina, testosterona, leptina y glicemia plasmática. ${ }^{49}$ También ha disminuido las tasas de aborto espontáneo a 8,8\% frente al placebo a $41,9 \%$, y las tasas de diabetes gestacional de 31\% a $3 \% .{ }^{41,50}$

- Dieta y ejercicio: existen muchas modalidades de actividad física como el tai-chi, viniyoga, pilates y spinning. Con la alimentación se debe alcanzar un peso saludable, aceptándose una pérdida entre 5\% y 10\% del peso; igualmente, una circunferencia abdominal menor a $80 \mathrm{~cm}$ en 6 meses es adecuada. ${ }^{41,51,52}$ De otra parte, aún no hay consenso generalizado sobre el uso del orlistat y/o sibutramina. En contraste, la cirugía bariátrica podría realizase en algunos casos. ${ }^{53}$

- Hirsutismo: se pueden utilizar métodos cosméticos como ceras y combinarlas con la electrólisis. También pueden emplearse los modificadores biológicos del folículo piloso, como el láser, constituyéndose en un método de depilación permanente. $^{35,54,55}$

Aunado a los métodos anteriores, se pueden utilizar antiandrógenos como espironolactona, ciproterona, flutamida, finasteride, ketoconazol, etc. Brian A, et ál, en un reciente metaanálisis, llegaron a la conclusión de que los antiandrógenos son agentes ligeramente eficaces para tratar el hirsutismo, teniendo mayor eficacia que la metformina y el placebo. Además, algunos parecen demostrar mejores resultados cuando se combinan con un anticonceptivo oral y la metformina. ${ }^{35,55,56}$

\section{Infertilidad}

El Consensus on Infertility Treatment Related to Polycystic Ovary Syndrome realizado en Grecia en 2007, recomendó en primera línea al citrato de clomifeno como inductor de la ovulación, seguido por la FSH recombinante o el Drilling laparoscópico, y finalmente, las técnicas de reproducción asistida. ${ }^{53}$

- Citrato de clomifeno: en dosis de 50 mg por día durante 5 días (dosis máxima de $150 \mathrm{mg}$ /día). El seguimiento folicular no es necesario, pero la gran mayoría de los centros hospitalarios a nivel mundial lo practica para hacer un reajuste de la dosis en ciclos posteriores. La ovulación ocurre entre $75 \%$ a $80 \%$ y las tasas de concepción son alrededor de 22\% por ciclo, es decir, 66\% en 3 ciclos para un total de 6 ciclos de tratamiento. Sin embargo, las tasas de concepción y ovulación aumentan en combinación con la metformina. ${ }^{41,53,57}$

- Gonatrofinas: se pueden utilizar dos protocolos: el "step down" y el "step up". No obstante, se prefiere este último por producir menor hiperestimulación ovárica y menos embarazos múltiples, por lo cual requiere seguimiento con ultrasonido. El régimen de bajas dosis produce ovulación monofolicular en el 70\%, embarazos entre el 15\% y $30 \%$ de los ciclos, y éxitos en tasas de embarazos de $62 \%$ en 6 ciclos. La duración del tratamiento es igualmente de 6 ciclos. ${ }^{41,53}$

- Drilling de ovarios por laparoscopia: es considerada una técnica aceptable para pacientes clomifeno-resistentes con resultados similares a las gonatrofinas. ${ }^{38,48}$

En contraste, cuando las anteriores terapias fracasan, se recurre a las técnicas de reproducción asistidas, circunstancia presente en las parejas con varias causas de infertilidad tal como la asociación de un SOP con factor tuboperitoneal y factor masculino severo. ${ }^{38,48}$ 


\section{CONCLUSIONES}

La fisiopatología y la etiología del exceso de andrógenos es muy variada y repercute negativamente a nivel estético, reproductivo y cardiovascular aunado a las alteraciones metabólicas. Por lo tanto, requiere un estudio y manejo multidisciplinario.

\section{AGRADECIMIENTOS}

A los doctores Ivonne Jeannette Díaz-Yamal, Luis Ernesto Pérez-Agudelo y Mike Jimmy CastañedaCastañeda por su colaboración en el servicio.

\section{REFERENCIAS}

1. Rodríguez Hierro F, Ibáñez Toda L. Hiperandrogenismo: diagnóstico. An Esp Pediatric 2002;56:28-33.

2. Martínez-García MA, Luque-Ramírez M, SanMillán JL, Escobar-Morreale HF. Body iron stores and glucose intolerance in premenopausal women: role of hyperandrogenism, insulin resistance, and genomic variants related to inflammation, oxidative stress, and iron metabolism. Diabetes Care 2009;32:1525-30.

3. Wiltgen D, Benedetto IG, Mastella LS, Spritzer PM. Lipid accumulation product index: a reliable marker of cardiovascular risk in polycystic ovary syndrome. Hum Reprod 2009;24:1726-31.

4. Biyasheva A, Legro R.S, Dunaif A, Urbanek M. Evidence for association between polycystic ovary syndrome (PCOS) and TCF7L2 and glucose intolerance in women with PCOS and TCF7L2. J Clin Endocrinol Metab 2009;94:2617-25.

5. Moran L, Teede H. Metabolic features of the reproductive phenotypes of polycystic ovary syndrome. Hum Reprod Update 2009;15:477-88.

6. Gambineri A, Pascuali R. Resistencia a la insulina, obesidad y síndrome metabólico en el síndrome de ovario poliquístico. Endocrinol Nutr 2006;53:41-5.

7. Ketel IJ, Stehouwer CD, Serne EH, Korsen TJ, Hompes PG, Smulders YM, et al. Obese but not normal-weight women with polycystic ovary syndrome are characterized by metabolic and microvascular insulin resistance. J Clin Endocrinol Metab 2008;93:3365-72.

8. Yildiz BO, Knochenhauer ES, Azziz R. Impact of obesity on the risk for polycystic ovary syndrome. J Clin Endocrinol Metab 2008;93:162-8.
9. Luque-Ramírez M, Mendieta-Azcona C, ÁlvarezBlasco F, Escobar-Morreale HF. Androgen excess is associated with the increased carotid intima-media thickness observed in young women with polycystic ovary syndrome. Hum Reprod 2007;22:3197-203.

10. Bhattacharya SM. Metabolic syndrome in females with polycystic ovary syndrome and International Diabetes Federation criteria. J Obstet Gynaecol Res 2008;34:62-6.

11. Sepilian V, Nagamani M. Adiponectin levels in women with polycystic ovary syndrome and severe insulin resistance. J Soc Gynecol Investig 2005;12:129-35.

12. Toulis KA, Goulis DG, Farmakiotis D, Georgopoulos NA, Katsikis I, Tarlatzis B, et al. Adiponectin levels in women with polycystic ovary syndrome: a systematic review and a meta-analysis. Hum Reprod Update 2009;15:297-307.

13. Hajer GR, van Haeften TW, Visseren FL. Adipose tissue dysfunction in obesity, diabetes, and vascular diseases. Eur Heart J 2008;29:2956-71.

14. Blank SK, McCartney CR, Chhabra S, Helm KD, Eagleson CA, Chang RJ, et al. Modulation of gonadotropinreleasing hormone pulse generator sensitivity to progesterone inhibition in hyperandrogenic adolescent girls--implications for regulation of pubertal maturation. J Clin Endocrinol Metab 2009;94:2360-6.

15. Gross KM, Matsumoto AM, Bremner WJ. Differential control of luteinizing hormone and follicle-stimulating hormone secretion by luteinizing hormone-releasing hormone pulse frequency in man. J Clin Endocrinol Metab 1987;64:675-80.

16. Spratt DI, Finkelstein JS, Butler JP, Badger TM, Crowley WF Jr. Effects of increasing the frequency of low doses of gonadotropin-releasing hormone (GnRH) on gonadotropin secretion in GnRH-deficient men. J Clin Endocrinol Metab 1987;64:1179-86.

17. Doi SA. Neuroendocrine dysfunction in PCOS: a critique of recent reviews. Clin Med Res 2008;6:47-53.

18. Liepa GU, Sengupta A, Karsies D. Polycystic ovary syndrome (PCOS) and other androgen excess related conditions: can changes in dietary intake make a difference? Nutr Clin Pract 2008;23:63-8.

19. Escobar-Morreale HF. Etiology of polycystic ovary syndrome. Endocrinol Nutr 2006;53:7-14.

20. Ehrmann D. Polycystic ovary syndrome. N Engl J Med 2005;352:1223-36.

21. Speroff L, Fritz M. Anovulación y poliquistosis ovárica. En: Speroff L, Fritz M, editores. Endocrinología 
ginecológica clínica y esterilidad. $7^{\mathrm{a}}$ ed. Barcelona (España): Wolters Kluwer Lippincott Williams \& Wilkings; 2007. p.465-98.

22. Shi D, Dyck M, Uwiera RR, Russell JC, Proctor SD, Vine DF. A unique rodent model of cardiometabolic risk associated with the metabolic syndrome and polycystic ovary syndrome. Endocrinology 2009;150:4425-36.

23. Queipo G. Hiperandrogenismo. Investiga 2007;3:6-7.

24. Satar N. PCOS, insulin resistance, and long-term risks for diabetes and vascular disease. Br J Diabetes Vasc Dis 2009;9:15-8.

25. Cornier MA, Dabelea D, Hernández TL, Lindstrom RC, Steig AJ, Stob NR, et al. The metabolic syndrome. Endocr Rev 2008;29:777-822.

26. Cho LW, Jayagopal V, Kilpatrick ES, Atkin SL. The mean and the biological variation of insulin resistance does not differ between polycystic ovary syndrome and type 2 diabetes. Ann Clin Biochem 2009;46:218-21.

27. Moran L, Teede H. Metabolic features of the reproductive phenotypes of polycystic ovary syndrome. Hum Reprod Update 2009;15:477-88.

28. Azziz R, Carmina E, Dewailly D, Diamanti-Kandarakis E, Escobar-Morreale HF, Futterweit W, et al. The Androgen Excess and PCOS Society criteria for the polycystic ovary syndrome: the complete task force report. Fertil Steril 2009;91:456-88.

29. Roa Barrios M, Arata-Ballarba G, Valeri L, VelásquezMaldonado E. Relationship between the triglyceride/ high-density lipoprotein-cholesterol ratio, insulin resistance index and cardiometabolic risk factors in women with polycystic ovary syndrome. Endocrinol Nutr 2009;56:59-65.

30. Pineda C. Síndrome metabólico: definición, historia, criterios. Colomb Med 2008;39:96-106.

31. Luque-Ramírez M, Mendieta-Azcona C, del Rey Sánchez JM, Matíes M, Escobar-Morreale F. Effects of an antiandrogenic oral contraceptive pill compared with metformin on blood coagulation tests and endothelial function in women with the polycystic ovary syndrome: influence of obesity and smoking. Eur J Endocrinol 2009;160:469-80.

32. Cascella T, Palomba S, De Sio I, Manguso F, Giallauria F, De Simone B, et al. Visceral fat is associated with cardiovascular risk in women with polycystic ovary syndrome. Hum Reprod 2008;23:153-9.

33. Kosmala W, O’Moore-Sullivan TM, Plaksej R, Kuliczkowska-Plaksej J, Przewlocka-Kosmala M,
Marwick TH. Subclinical impairment of left ventricular function in young obese women: contributions of polycystic ovary disease and insulin resistance. J Clin Endocrinol Metab 2008;93:3748-54.

34. Hosseinian AH, Kim MH, Rosenfield RL. Obesity and oligomenorrhea are associated with hyperandrogenism independent of hirsutism. J Clin Endocrinol Metab 1976;42:765-9.

35. Swingler R, Awala A, Gordon U. Hirsutism in young women. The Obstetrician \& Gynaecologist 2009;11:101-7.

36. Castelo-Branco C, Peralta S. Hiperandrogenismo ovárico. Valoración clínica y terapéutica. Clin Invest Ginecol Obstet 2005;32:244-56.

37. d'Alva CB, Abiven-Lepage G, Viallon V, Groussin L, Dugue MA, Bertagna S, et al. Sex steroids in androgensecreting adrenocortical tumors: clinical and hormonal features in comparison with non-tumoral causes of androgen excess. Eur J Endocrinol 2008;159:641-7.

38. Rosenfield RL. Clinical practice. Hirsutism. N Engl J Med 2005;353:2578-88.

39. Yildiz BO, Bolour S, Woods K, Azziz R. Visually scoring hirsutism. Hum Reprod Update 2009; [Epub ahead of print].

40. Ferriman D, Gallwey JD. Clinical assessment of body hair growth in women. J Clin Endocrinol Metab 1961;21:1440-7.

41. Guías Consenso Colombiano Síndrome de Ovario Poliquístico. Bogotá: Grupo de Endocrinología Ginecológica-FUNCOBES; 2007.

42. Libè A, Fratticci A, Bertherat J. Adrenocortical cancer: pathophysiology and clinical management. Endocr Relat Cancer 2007;14 13-28.

43. Cools M, Drop SL, Wolffenbuttel KP, Oosterhuis JW, Looijenga LH. Germ cell tumors in the intersex gonad: old paths, new directions, moving frontiers. Endocr Rev 2006;27:468-84.

44. Nader S, Diamanti-Kandarakis E. Polycystic ovary syndrome, oral contraceptives and metabolic issues: new perspectives and a unifying hypothesis. Hum Reprod 2007;22:317-22.

45. Hoeger K, Davidson K, Kochman L, Cherr y T, Kopin L, Guzick DS. The impact of metformin, oral contraceptives, and lifestyle modification on polycystic ovary syndrome in obese adolescent women in two randomized, placebo-controlled clinical trials. J Clin Endocrinol Metab 2008;93:4299-306. 
46. Palomba S, Falbo A, Orio F Jr, Manguso F, Russo T, Tolino A, et al. A randomized controlled trial evaluating metformin pre-treatment and co-administration in non-obese insulin-resistant women with polycystic ovary syndrome treated with controlled ovarian stimulation plus timed intercourse or intrauterine insemination. Hum Reprod 2005;20:2879-86.

47. Jensterle M, Janez A, Mlinar B, Marc J, Prezelj J, Pfeifer M. Impact of metformin and rosiglitazone treatment on glucose transporter 4 mRNA expression in women with polycystic ovary syndrome. Eur J Endocrinol 2008;158:793-801.

48. Moll E, Korevaar JC, Bossuyt PM, van der Veen F. Does adding metformin to clomifene citrate lead to higher pregnancy rates in a subset of women with polycystic ovary syndrome? Hum Reprod 2008;23:1830-4.

49. Nelson S. Obesity, insulin resistance and assisted conception The British Journal of Diabetes \& Vascular Disease 2009;9:23-6.

50. Palomba S, Falbo A, Zullo F, Orio F Jr. Evidence-based and potential benefits of metformin in the polycystic ovary syndrome: a comprehensive review. Endocr Rev 2009;30:1-50.

51. Thomson RL, Buckley JD, Noakes M, Clifton PM, Norman R, Brinkworth GD. The effect of a hypocaloric diet with and without exercise training on body composition, cardiometabolic risk profile, and reproductive function in overweight and obese women with polycystic ovary syndrome. J Clin Endocrinol Metab 2008;93:3373-80.

52. Pasquali R, Antenucci D, Casimirri F, Venturoli S, Paradisi R, Fabbri R, et al. Clinical and hormonal characteristics of obese amenorrheic hyperandrogenic women before and after weight loss. J Clin Endocrinol Metabs 1989;68:173-9.

53. Consensus on infertility treatment related to polycystic ovary syndrome. The Thessaloniki ESHRE/ASRMSponsored PCOS Consensus Workshop Group. Hum Reprod 2008;23:462-77.

54. Koulouri O, Conway G. Management of hirsutism. BMJ 2009;338:b847.

55. Martin KA, Chang RJ, Ehrmann DA, Ibanez L, Lobo RA, Rosenfield RL, et al. Evaluation and treatment of hirsutism in premenopausal women: an Endocrine Society clinical practice guideline. J Clin Endocrinol Metab 2008;93:1105-20.

56. Swiglo BA, Cosma M, Flynn DN, Kurtz DM, Labella ML, Mullan RJ, et al. Clinical review: antiandrogens for the treatment of hirsutism: a systematic review and metaanalyses of randomized controlled trials. J Clin Endocrinol Metab 2008;93:1153-60.

57. Moll E, van der Veen F, van Wely M. The role of metformin in polycystic ovary syndrome: a systematic review. Hum Reprod Update 2007;13:527-37. 